\title{
On the oscillation spectrum of a magnetized core in a giant star
}

\author{
Michel Rieutord ${ }^{1,2, \star}$ \\ ${ }^{1}$ Université de Toulouse; UPS-OMP; IRAP; Toulouse, France \\ ${ }^{2}$ CNRS; IRAP; 14, avenue Edouard Belin, F-31400 Toulouse, France
}

\begin{abstract}
The spectrum of gravito-acoustic modes is depleted in dipolar modes for a significant fraction of the giant stars observed by the Kepler mission, a feature that has been explained by the presence of magnetic fields in the core of these stars (Fuller et al. 2015, Cantiello et al. 2016). We further investigate this possible scenario by considering first the oscillation spectrum of the core of a giant star modeled by a stably stratified, self-gravitating fluid of uniform density in a sphere pervaded by a uniform magnetic field. Our results show that the first effect of a magnetic field on the g-modes is to reduce their wavenumber and therefore reduce their damping. The magnetic effect, on this model, is therefore opposite Fuller's et al scenario. Moreover, the model shows that it is not possible to change the damping rate without changing the frequency of the modes and this latter change is not observed. Because of the simplicity of our model, the magnetized core scenario cannot be dismissed but further investigations are needed, and other ways of explaining the presence of depressed modes should also be considered.
\end{abstract}

\section{Introduction}

One of the important results obtained by the Kepler mission as far as asteroseismology is concerned, is the detection of mixed acoustic gravity modes in giant stars [1]. The detection of mixed acoustic-gravity modes is indeed a real opportunity to obtain informations on the core of giant stars and more generally on the core of stars. A particularly puzzling result of these observations has been the relatively slow rotation of the core when compared to the expected values given by models [2,3]. Clearly data show that the mechanism, which controls the exchange angular momentum between a core and an envelope of a star, is not correctly implemented in stellar evolution models, if at all correctly understood.

However, the mysteries of the giant stars do not stop here. Seismic observations also show that a significant fraction of giant stars do not show all the modes they should. Dipolar $(\ell=1)$ modes are often depleted, with amplitudes strongly varying from one star to the next [1], while $\ell=2$ or $\ell=3$ modes are only slightly or not depleted [4]. Since the modes are most likely excited stochastically by the turbulent convection in the envelope (like solar acoustic modes), the selection of visible modes may be the result of some damping mechanism. Fuller et al. [5] suggested that magnetic fields in the radiative core could play an important role in this selection process. The idea is that g-modes of the core, which couple to p-modes of the envelope to make the mixed modes, are presumably strongly damped by their interaction with a residual magnetic field trapped in the core. While $\ell=1$ modes are

\footnotetext{
^e-mail: mrieutord@irap.omp.eu
}

damped by such a mechanism, $\ell=2$ (or higher) are much less affected because of a weaker coupling between their gravity and acoustic parts.

The scenario imagined by Fuller et al. [5] is appealing as it opens a window on the magnetic history of giant stars and more generally on dynamos in convective cores. However, these results are based on a local (WKB) analysis of the waves and assume that waves entering a magnetized core are fully dissipated. Hence, an open question is whether such a scenario is confirmed by a global analysis that takes the whole star into account. If confirmed, global inversion of the data may open a new window on the magnetic activity of convective core and on the dynamics history of intermediate mass stars. The road towards such a global model is however not a piece of cake since many parts of the problem are ill-known. In particular the way g-modes interact with magnetic fields has not been studied very much.

To progress on this question we consider a simplified model of the core of a giant star and study the effects of a uniform magnetic field on the damping rates of the gmodes. Results are surprising at first sight since magnetized g-modes are less damped than the non-magnetized ones. Such results are easily explained by the structure of the magnetized g-modes but they tease us to more in depth studies.

The paper is organized as follows: in the following section we give all the details of our simple model. In section 3 we give the results of the first investigations focusing on the modes that have the right frequency range. Some preliminary conclusions follow. 


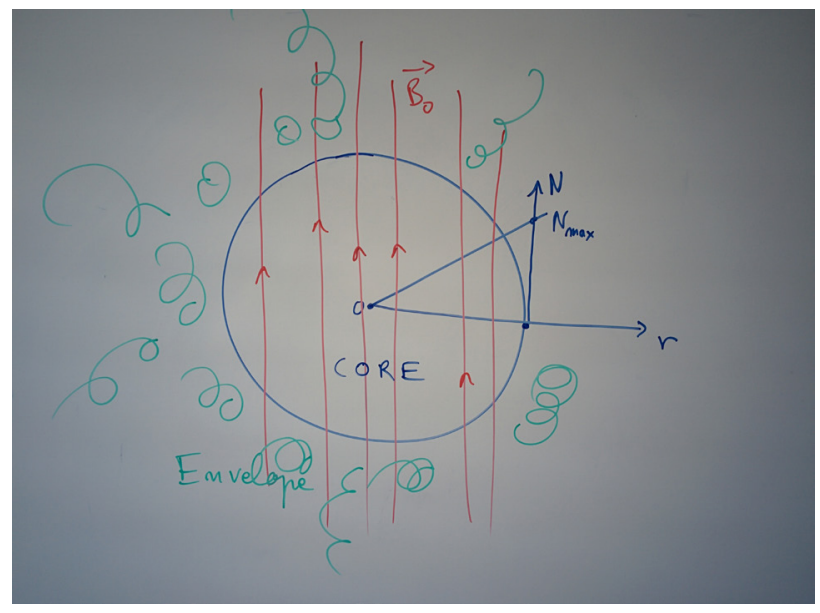

Figure 1: Schematic view of the simplified model of the magnetized radiative core of a giant star.

\section{The simplified model}

We consider an quasi-incompressible fluid filling a sphere of radius $R$, where cold sources are distributed, and bathed by a uniform magnetic field $\vec{B}_{0}=B_{0} \vec{e}_{z}$ as sketched out in Fig.1. Cold sources, unlike heat sources, generate a stably stratified fluid where gravity modes can propagate. The equilibrium temperature field $T_{\text {eq }}$ thus verify

$$
\chi \Delta T_{\text {eq }}+Q=0
$$

where $\chi$ is the heat conductivity and $Q<0$. Solving with imposed spherical symmetry leads to

$$
T_{\text {eq }}=-\frac{Q r^{2}}{6 \chi}+T_{c}
$$

where $r$ is the radial coordinate and $T_{c}$ the central temperature. The perturbed momentum and heat (linearized) equations read

$$
\begin{gathered}
\frac{\partial \vec{v}}{\partial t}=-\vec{\nabla} \delta P / \rho+\frac{\delta \rho}{\rho} \vec{g}+v \Delta \vec{v}+\overrightarrow{\operatorname{Rot}} \vec{b} \times \frac{\vec{B}_{0}}{\rho \mu_{0}} \\
\frac{\partial \delta T}{\partial t}+(\vec{v} \cdot \vec{\nabla}) T_{\mathrm{eq}}=\kappa \Delta \delta T
\end{gathered}
$$

where $\vec{v}, \vec{b}, \delta P$ and $\delta T$ are the velocity, magnetic field, pressure and temperature disturbances, respectively. $\rho$ is the density, $v$ the kinematic viscosity, $\kappa$ the heat diffusivity, $\mu_{0}$ the vacuum permittivity and $\vec{g}$ the gravity. Obviously, we shall use the Boussinesq approximation, so mass conservation implies

$$
\operatorname{Div} \vec{v}=0
$$

We now rewrite the whole system of equations with scaled quantities, using the radius of the sphere $R$ as the length scale, the inverse of the maximum Brunt-Väisälä frequency as the time scale, $|Q| R^{2} / \chi$ as the temperature scale and $B_{0}$ as the magnetic field scale. The Brunt-Väisälä frequency reads

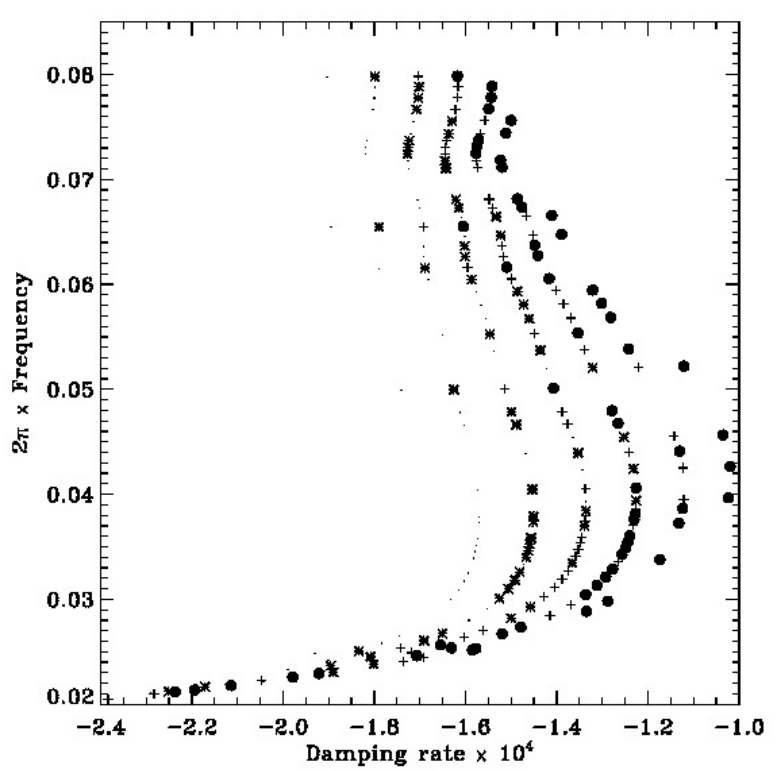

Figure 2: Evolution of four eigenvalues around $\omega=0.01 \mathrm{~N}$ when $10^{-8} \lesssim \mathcal{A} \lesssim 10^{-4}$. Here, $E_{v}=10^{-10}, E_{\eta}=10^{-10}$ and $E_{t}=10^{-7}$.

$$
N(r)=N r \quad \text { with } \quad N^{2}=\frac{\alpha|Q| R g_{s}}{3 \chi}
$$

where $r$ is the scaled radial coordinate, $\alpha$ the isobaric dilation coefficient and $g_{s}$ the surface gravity. As the Boussinesq approximation is used $\vec{g}=-g_{s} \vec{r}$. Thus doing, we find

$$
\begin{gathered}
\vec{\nabla} \cdot \vec{u}=\vec{\nabla} \cdot \vec{b}=0 \\
\partial_{\tau} \vec{b}=\vec{\nabla} \times\left(\vec{u} \wedge \vec{e}_{z}\right)+E_{\eta} \Delta \vec{b} \\
\partial_{\tau} \theta+r u_{r}=E_{t} \Delta \theta \\
\partial_{\tau} \vec{u}=-\vec{\nabla} p+\theta \vec{r}+E_{v} \Delta \vec{u}+\mathcal{A}(\vec{\nabla} \times \vec{b}) \times \vec{e}_{z}
\end{gathered}
$$

where we set

$$
\mathcal{A}=\left(\frac{V_{a}}{N R}\right)^{2}, \quad E_{v}=\frac{v}{N R^{2}}, \quad E_{t}=\frac{\kappa}{N R^{2}}, \quad E_{\eta}=\frac{\eta}{N R^{2}}
$$

Hence, the problem depends on three diffusion parameters $\left(E_{v}, E_{t}, E_{\eta}\right)$ and the coupling constant between gravity modes and Alfvén waves $\mathcal{A}$. With typical parameters of giant cores, we find that

$$
E_{v} \sim 10^{-18}, \quad E_{\eta} \sim 10^{-18}, \quad E_{t} \sim 10^{-11}
$$

Viscosity and magnetic diffusion are therefore extremely weak, and heat diffusion remains the basic source of damping. Since such numbers are not reachable numerically, we choose to consider higher values, namely

$$
E_{v} \sim 10^{-10}, \quad E_{\eta} \sim 10^{-10}, \quad E_{t} \sim 10^{-7}
$$



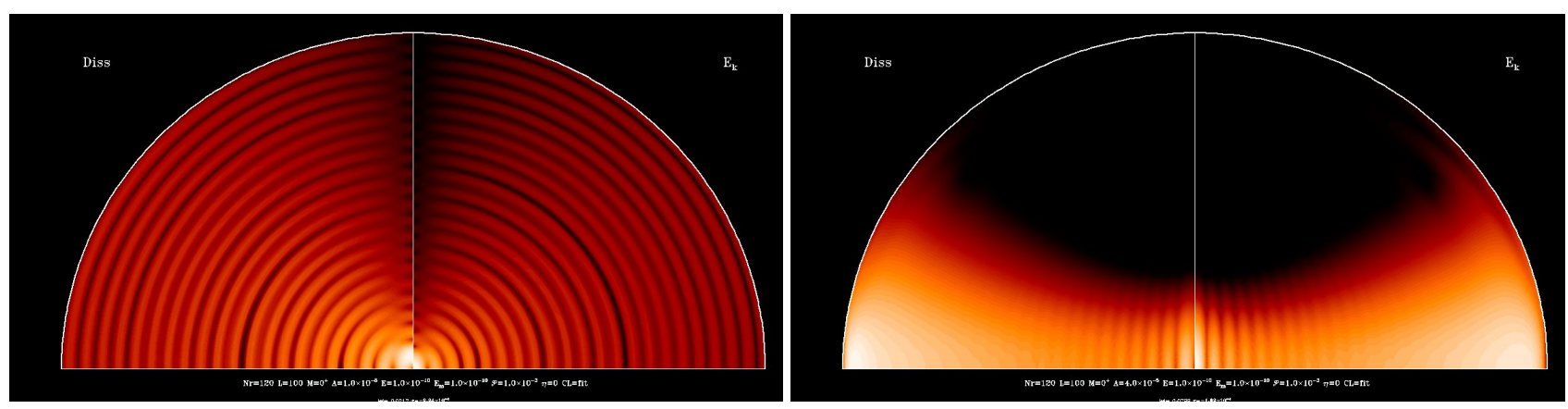

Figure 3: Left: Eigenfunction when the magnetic field is weak $\left(\mathcal{A}=10^{-8}\right)$. Right: Eigenfunction when the magnetic field is strong $\left(\mathcal{A}=4 \times 10^{-5}\right)$. On both plots we show on the right side the kinetic energy of the mode, while on the left side we show the viscous dissipation.

However, these higher values do not changed the qualitative results. [5] suggest that the maximum value of the Brunt-Väisälä frequency in the core is around $10^{4} \mu \mathrm{Hz}$. As for the magnetic field intensity we also follow [5] and take

$$
2 \times 10^{5} \mathrm{G} \lesssim B_{0} \lesssim 2 \times 10^{7} \mathrm{G}
$$

Taking a typical core radius of a $1.5 \mathrm{M}_{\odot}$-giant of $4.2 \times 10^{8} \mathrm{~m}$, and a mean density of $1.6 \mathrm{~g} / \mathrm{cm}^{3}$ (from a MESA model [6]), we find that

$$
10^{-8} \lesssim \mathcal{A} \lesssim 10^{-4}
$$

so rather small values for the coupling coefficient.

\section{First results}

We solve the set of equations (7) by first assuming an exponential time-dependence, namely all perturbations are $\propto \exp (\lambda \tau)$ where $\lambda$ is either the complex eigenvalue (free modes) or the complex frequency ( $i \omega$ for forced oscillations). Then we discretize the partial differential equations on a spectral grid using spherical harmonics expansions, namely

$$
\vec{u}=\sum_{l=0}^{+\infty} \sum_{m=-l}^{+l} u_{m}^{\ell}(r) \vec{R}_{\ell}^{m}+v_{m}^{\ell}(r) \vec{S}_{\ell}^{m}+w_{m}^{\ell}(r) \vec{T}_{\ell}^{m}
$$

with

$$
\vec{R}_{\ell}^{m}=Y_{\ell}^{m}(\theta, \varphi) \vec{e}_{r}, \quad \vec{S}_{\ell}^{m}=\vec{\nabla} Y_{\ell}^{m}, \quad \vec{T}_{\ell}^{m}=\vec{\nabla} \times \vec{R}_{\ell}^{m}
$$

as in $[7,8]$. Examples of the projected equations on the spherical harmonics basis may be found in [9-11]. The radial functions are then discretized on the Gauss-Lobatto grid leading to a generalized eigenvalue problem for the magneto-gravity modes (see Valdettaro et al. [12]).

\subsection{Free modes}

The typical frequency of the depleted mixed modes is around $100 \mu \mathrm{Hz}$ so we need to consider gravity modes of frequency around $10^{-2} \mathrm{~N}$. We therefore monitored 4 eigenvalues associated with gravity modes with $\omega \sim 2 \times 10^{-2}$ for $B_{0}=0$, when the coupling parameter $\mathcal{A}$ is progressively increased.

We show in Fig. 2 the evolution of eigenvalues in the complex plane when $10^{-8} \lesssim \mathcal{A} \lesssim 10^{-4}$. From this plot we see that the damping rate first diminishes while the frequency slighly increases. Then, when $\mathcal{A} \gtrsim 1.1 \times 10^{-6}$, the damping rate approximately saturates, while the frequency continuously increases.

Hence, the first effect of magnetic fields is to reduce the damping of gravity modes. This is easily understood if we have a look at the eigenfunctions with and without magnetic fields. A meridional view of the eigenmodes is shown in Fig. 3. We note that at a frequency of a few percent of the Brunt-Väisälä frequency, $\ell=1$-g-modes have a high radial wavenumber. On the contrary, in the low frequency range, Alfvén modes have a low wavenumber. The mixed magneto-gravity modes make a compromise between their double nature and show a typical wavenumber that is lower than their equivalent pure g-mode. Thus, the resulting wavenumber is decreased and the damping rate is decreased as well.

\subsection{Forced modes}

To view the impact of magnetic fields on g-modes from a different side, we also consider magneto-gravity modes forced by a deformation of the boundary. We also choose a frequency of $\sim 0.01 \mathrm{~N}$ and now monitor the viscous dissipation as the strength of the magnetic field is increased. Such a forcing mimics the coupling between the acoustic modes of the envelope and the magneto-gravity modes of the core. The result is shown in Fig. 4 for two values of the parameter $E_{v}$. In the two cases we note that the viscous dissipation decreases as $B_{0}$ increases, thus confirming the trend observed on free modes.

\section{Discussion and conclusions}

Alfvén modes are high frequency modes whose lowest frequency is of the order of $V_{A} / R, V_{A}$ being the Alfvén speed and $R$ the radius of the core. Besides, gravity modes are 


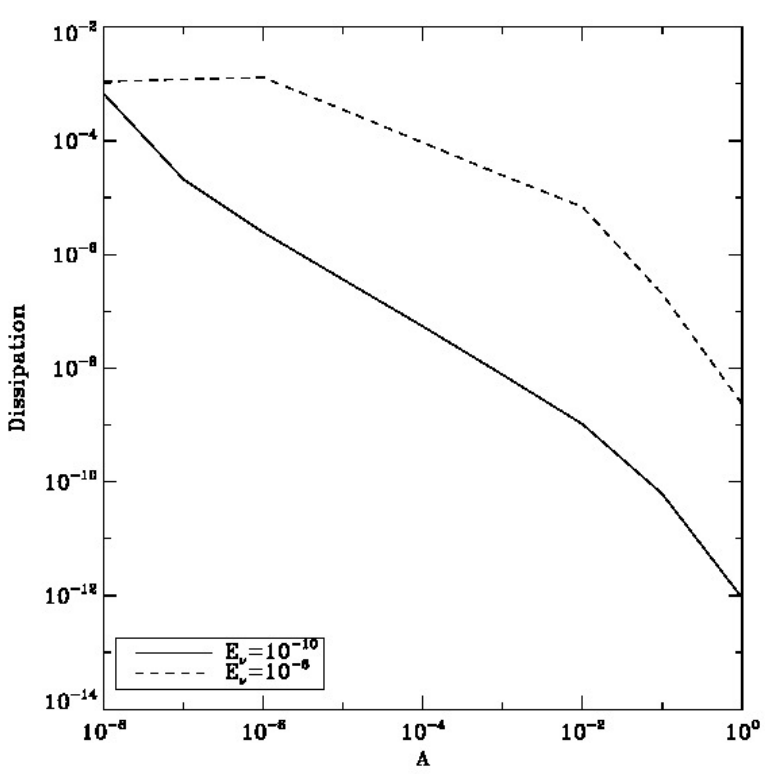

Figure 4: Evolution of the viscous dissipation with the coupling parameter $\mathcal{A}$ for $E_{v}=10^{-6}$ and $E_{v}=10^{-10}$.

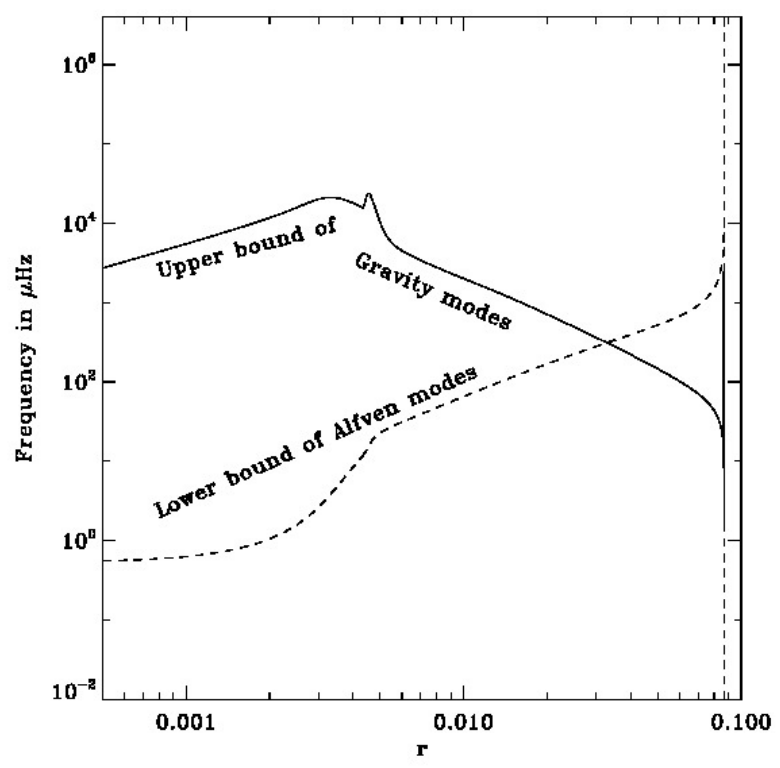

Figure 5: Propagation region for gravity and Alfvén waves in the core of giant star of mass $1.5 \mathrm{M}_{\odot} . r$ is the radial coordinate scaled by the star radius $\mathrm{R}_{*}=4.87 \times 10^{11} \mathrm{~cm}$.

low frequency modes whose highest frequency is bounded by the Brunt-Väisälä frequency. For both of them, when the frequency is close to the limiting frequency, the wavelength is the largest possible. Magneto-gravity modes, for which magnetic field and stratification are both important, are thus expected to be a combination of long wavelength Alfvén and gravity modes as illustrate in Fig. 5. Such magneto-gravity modes should not therefore be very good at dissipating energy. The simplified Boussinesq model used to investigate the properties of such modes is indeed confirming that g-modes perturbed by a magnetic field have a reduced damping rate in the first place compared to the unperturbed modes.

One may argue that a uniform magnetic field might not be very realistic to represent magnetic fields in the core of giant stars. [5] indeed suggest that a highly disordered magnetic field is inducing the so-called magnetic greenhouse effect. The difficulty is that during the period between the end of the main sequence, where a dynamo could operate in the core, and the giant stage that is observed, small-scale magnetic fields are dissipated. For a typical $1.5 \mathrm{M}_{\odot}$ giant star, the time lapse is $\sim 700$ Myrs. This is larger than the diffusion time of all scales larger than $0.035 R_{\text {core }}$. Moreover dynamos tend to build-up largescale magnetic fields (e.g. Brandenburg et al.[13]). This let us think that even if the field is not uniform, it is expected to be dominated by the large-scales, in which case the magnetic perturbations should act in the same way as with our uniform field.

Using an approach based solely on observational data, Mosser et al. [14] recently showed that depressed modes are mixed modes, implying that the resonant cavity is similar for stars with and without depressed modes. Except the amplitudes, the spectrum of stars with depressed modes show no difference with the one with non-depressed modes. Hence, in view of the results of the foregoing model, it seems difficult to use magnetic fields to damp dipole modes without perturbing the frequencies as imposed by observations.

This does not exclude magnetic fields from being at the origin of the effect, but it is then a more subtle effect than just being an absorber. Our model is certainly very crude and one can think about the effect of density variations on the magnetic side of the magneto-gravity modes. Indeed, in the core of a giant the density varies by orders of magnitude. This leads to strong variations of the Alfvén speed that will modify the properties of the mixed magneto-gravity modes. This subject remains to be investigated.

Another possibility that should be studied is a mechanism that weakens the excitation of the modes. Such a mechanism would easily explain the point made by Mosser et al. (2016), but it remains to be found.

\section{Acknowledgements}

I would like to warmly thank Sébastien Deheuvels for drawing my attention to this problem and for enlightening discussions on the properties of giant stars.

\section{References}

[1] B. Mosser, Y. Elsworth, S. Hekker, D. Huber, T. Kallinger, S. Mathur, K. Belkacem, M.J. Goupil, R. Samadi, C. Barban et al., A\&A 537, A30 (2012)

[2] S. Deheuvels, R.A. García, W.J. Chaplin, S. Basu, H.M. Antia, T. Appourchaux, O. Benomar, G.R. Davies, Y. Elsworth, L. Gizon et al., ApJ 756, 19 (2012) 
[3] P. Eggenberger, J. Montalbán, A. Miglio, A\&A 544, L4 (2012)

[4] D. Stello, M. Cantiello, J. Fuller, R.A. Garcia, D. Huber, Pub. Astron. Soc. Austr. 33, e011 (2016)

[5] J. Fuller, M. Cantiello, D. Stello, R.A. Garcia, L. Bildsten, Science 350, 423 (2015)

[6] B. Paxton, L. Bildsten, A. Dotter, F. Herwig, P. Lesaffre, F. Timmes, Astrophys. J. Supp. Ser. 192, 3 (2011)

[7] M. Rieutord, Geophys. Astrophys. Fluid Dyn. 39, 163 (1987)

[8] M. Rieutord, L. Valdettaro, J. Fluid Mech. 341, 77 (1997)

[9] F. Rincon, M. Rieutord, A\&A 398, 663 (2003)
[10] D. Reese, F. Rincon, M. Rieutord, A\&A 427, 279 (2004)

[11] S. Abbassi, M. Rieutord, V. Rezania, MNRAS 419, 2893 (2012)

[12] L. Valdettaro, M. Rieutord, T. Braconnier, V. Fraysse, J. Comput. and Applied Math. 205, 382 (2007), physics/0604219

[13] A. Brandenburg, D. Sokoloff, K. Subramanian, Space Science Rev. 169, 123 (2012)

[14] B. Mosser, K. Belkacem, C. Pincon, M. Takata, M. Vrard, C. Barban, M. Goupil, T. Kallinger, R. Samadi, to appear in A\&A, ArXiv eprints/161003872 (2016) 